\title{
LA CONSULTA DE TAROT EN SANTIAGO DE CHILE: RASGOS SITUACIONALES Y DISCURSIVOS
}

\author{
TAROT READING IN SANTIAGO, CHILE: SITUATIONAL AND DISCURSIVE
}

\section{Claudia Abarca Cea}

Universidad Tecnológica Metropolitana

\section{Resumen}

Este artículo constituye una propuesta de descripción de la consulta de tarot en Santiago de Chile, un tipo de discurso interaccional hasta ahora nada estudiado en el ámbito de la lingüística, a partir de los rasgos situacionales y discursivos que le son propios: el marco de interacción, los participantes, la relación que se establece entre el consultante y el lector de cartas, la planificación de la consulta, el dinamismo comunicativo, la finalidad transaccional argumentativo-persuasiva y la modalidad lingüística empleada.

El análisis, que sigue la propuesta de variación diafásica de Briz (2010), está basado en un corpus formado por interacciones entre tarotistas y consultantes, así como en datos obtenidos de clases presenciales de tarot, páginas electrónicas, etc. Cabe señalar la novedad de dicho corpus en el panorama de los estudios lingüísticos

PALABRAS ClAVE: consulta de tarot, discurso, argumentación.

\begin{abstract}
This article analyzes tarot readings in Santiago, Chile. The aim is to describe a type of interactional discourse in the field of Linguistics that has not yet been studied. This description covers situational and discursive characteristics. It considers its interactional framework, participants, the relationship established between the client and the tarot reader, tarot reading planning, its communicative dynamism, argumentative-persuasive transactional purpose and the linguistic modality used.
\end{abstract}

This analysis follows the diaphasic variation proposal by Briz (2010) and is based on a corpus that includes interactions between tarot readers and clients, as well as data obtained from face-toface tarot classes and websites, among others. It is important to note the novelty of this study in the field of Linguistics.

KEY WORDS: tarot reading, speech, argumentation. 


\section{PRESENTACIÓN}

Diversas culturas han usado instrumentos para conocer el futuro, aclarar cuestiones dudosas del pasado u orientarse antes de tomar decisiones. Así, en Europa Occidental, se adoptaron sistemas de consultas, como la cartomancia, especialmente el tarot (del fr. "tarot", y este del it. "tarocco"), "una baraja formada por 78 naipes que llevan estampadas diversas figuras" (RAE, 2009).

Este artículo presenta un estudio, de tipo descriptivo-cualitativo, que se propone caracterizar, la consulta de tarot ${ }^{1}$ en la ciudad de Santiago de Chile, a partir de propuesta de variación diafásica de Briz (2010). Con el fin de cumplir este objetivo, se recogió información para obtener una muestra representativa de los tarotistas residentes 20 años o más en esta ciudad. Por la ausencia de estudios acerca del perfil sociológico de los lectores de cartas, en 2013 aplicamos una encuesta cuyo resultado fue un universo de 135 tarotistas.

La aplicación de la técnica de muestreo estratificado, basado en la metodología de Briz y Val. Es.Co. (2002), dio como resultado una muestra de 24 tarotistas, que representan estratos de la población según sexo (hombre $(H) /$ mujer $(M)$ ), edad $(26-55 />55)^{2}$, nivel sociocultural (alto y medio3). Como refleja el siguiente cuadro, las mujeres representan el $62 \%$ y el grupo más numeroso corresponde a lectoras con mayor instrucción de 25 a 55 años, adhesión que coincide con estudios realizados en Chile, como indicaremos en los datos sociohistóricos:

\begin{tabular}{|c|c|c|}
\hline \multicolumn{3}{|c|}{ MUESTRA DE TAROTISTAS } \\
\hline SEXO & NIVEL ALTO & NIVEL MEDIO \\
\hline \multirow{2}{*}{ MUJER } & $25-55: 6(25 \%)$ & $25-55: 3(12,5 \%)$ \\
\cline { 2 - 3 } & $>55: 4(16 \%)$ & $>55: 2(8,33 \%)$ \\
\hline \multirow{2}{*}{ HOMBRE } & $25-55: 3(12,5 \%)$ & $25-55: 2(8,33 \%)$ \\
\cline { 2 - 3 } & $>55: 2(8,33 \%)$ & $>55: 2(8,33 \%)$ \\
\hline
\end{tabular}

\footnotetext{
1 En este artículo, se presentan algunos resultados obtenidos en la tesis doctoral realizada en la Universidad de Valladolid "La consulta de tarot en Chile. Análisis de las estrategias discursivas empleadas por los tarotistas", dirigida por el Dr. Antonio Briz.

${ }^{2}$ Como ocurre en el universo total de tarotistas, no se presenta el estrato generacional de 18 a 25 años.

${ }^{3}$ En nuestro estudio, obtuvimos información de tarotistas de nivel alto y medio, esto es, solo con estudios superiores y secundarios. Para comprender este hecho, se debe considerar que en la Región Metropolitana, donde se ubica la ciudad de Santiago, el 93,6\% neto de su población cursó la enseñanza básica (SERPLAC, 2009).
} 
El corpus analizado, de aproximadamente 28 horas, corresponde a 28 lecturas desarrolladas cara a cara ${ }^{4}$. y grabadas entre los años 2011 y 2015. Los consultantes son de ambos sexos, de 22 a 59 , y con instrucción media y alta. ${ }^{5}$

Para la caracterización del evento nos servimos también de datos obtenidos en libros de tarot, en páginas electrónicas que ofrecen el servicio, así como en el registro de la observación participante de cuatro clases de tarot de aproximadamente dos horas cada una, dictadas durante los años 2013 y 2014. Además, realizamos preguntas adicionales a los hablantes de la muestra, con el fin de obtener información sobre el ejercicio profesional.

En este artículo, en primer lugar, entregamos antecedentes sociohistóricos del tarot. En segundo lugar, según datos proporcionados por tarotistas santiaguinos y las fuentes indicadas, describimos los rasgos situacionales y discursivos. Se caracteriza así el marco espacial, los participantes de la interacción, el dinamismo comunicativo, la planificación de la sesión, la finalidad del evento y la modalidad lingüística.

\section{EL TAROT: ANTECEDENTES SOCIOHISTÓRICOS}

El tarot es un naipe de origen desconocido a cuyos símbolos se les adjudica la capacidad de reflejar información relevante para el ser humano. Para Agar (2011:20), consultar esta baraja responde a un fenómeno propio de la posmodernidad "un retorno a las tradiciones del pasado, incluso al tribalismo, como resultados del proceso globalizador; una sinergia entre las formas arcaicas y el desarrollo tecnológico". Se trata, según Parker (2008), de nuevos códigos de disidencia religiosa en relación con lo oficial, una subversión ética del orden moral imperante. El sujeto religioso busca lo esotérico, debido a que esos símbolos complejos lo nutren de nuevas realidades opuestas a la materialidad del consumo, de compromisos históricos y del exceso de información.

En América Latina, además, en los sectores medios, especialmente desde 1980, se produce la expansión de la "Nueva Era": un conjunto de creencias diversas que puede claramente asociarse a la postmodernidad por las nociones de autonomía individual y fluidez (Míguez en Bahamondes 2000:112-113).

En Chile, la lectura de tarot es legal desde el año 2003. Según estudios del Instituto Nacional de la Juventud (2006 y 2009), alrededor de un $20 \%$ de jóvenes cree en el tarot, con una mayor aceptación de mujeres con mayor instrucción, tal como se refleja en nuestra muestra. Este porcentaje de adhesión también se obtiene en una encuesta

\footnotetext{
${ }^{4}$ No obtuvimos cooperación de tarotistas que atienden por medios electrónicos.

${ }^{5}$ La investigadora no logró contactar a consultantes de nivel bajo de instrucción. Como señalamos, en Santiago, la mayoría de la población de jóvenes y adultos ha cursado la enseñanza básica.
} 
nacional aplicada durante los años 2015 y 2016 sobre la forma en que los chilenos entienden la ciencia y la actividad científica (CONICYT, 2016).

Actualmente, en el país hay escuelas que enseñan a leer el tarot ${ }^{6}$, una amplia oferta del servicio por distintos medios de difusión, programas de televisión y de radio que incorporan tarotistas en sus paneles o concursos, diarios y revistas que publican predicciones basadas en la baraja y espacios públicos cedidos para ejercer el oficio.

\section{LA CONSULTA DE TAROT: RASGOS SITUACIONALES Y DISCURSIVOS}

La consulta de tarot está determinada por un ámbito concreto, la lectura de símbolos que revelan aspectos de la vida del consultante, y por unos profesionales que ofrecen unos servicios de interpretación de cartas por los que cobran.

El ámbito en que se inscribe la cartomancia es el conocimiento esotérico, que alude a lo oculto, reservado. Habitualmente, la consulta responde a un plan predeterminado en un tiempo cercano a una hora y su valor puede alcanzar cerca de 80 dólares. Si el consultante lo desea, la sesión puede ser grabada o apuntada. Según los datos de nuestra muestra, los lectores prefieren los tarots tradicionales denominados Marsella y Rider Waite.

Asimismo, existen tarotistas que añaden otros servicios a la consulta. Moulian (2000) los identifica como agentes mágicos, caras visibles de un universo mistérico. En un estudio realizado en Chile, señala que predominan los que, junto con leer el tarot, prometen alejar el mal, encontrar objetos perdidos y solucionar problemas amorosos o sexuales. Actualmente, tal como aparece en estos avisos, la consulta, además, aparece en la oferta de terapias alternativas:
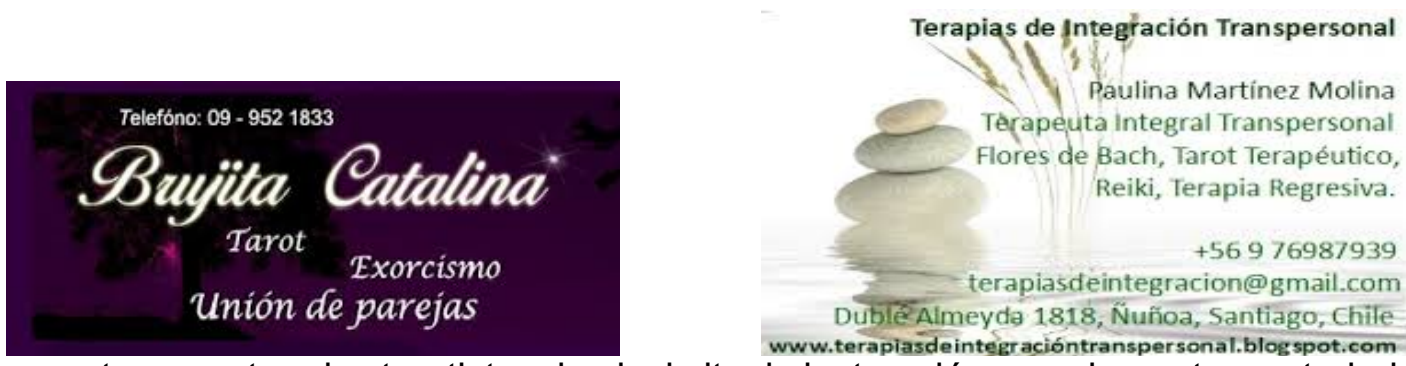

En nuestra muestra, dos tarotistas de nivel alto de instrucción complementan su trabajo de terapia psicológica con la lectura de tarot

6 Syncronia, Círculo Aleph, Instituto Saint Germain, Centro Onahi, entre otros.

7 Búsqueda a través de www.google.com con fecha 18 de febrero de 2018. 
A continuación, siguiendo la propuesta de variación diafásica de Briz (2010), describiremos este discurso interaccional según sus rasgos situacionales y discursivos.

\subsection{El marco espacial}

La consulta de tarot es una interacción oral que se realiza cara a cara, en un lugar denominado consulta. También se realiza a través de medios electrónicos (internet, teléfono, radio y televisión). Como en la conversación telefónicas, en esta segunda modalidad, aunque sí hay simultaneidad temporal (adecuación entre el tiempo de emisión y el de recepción), no existe copresencia de los interlocutores.

En nuestra muestra, la consulta se ubica en un sitio público (plazas, calles y paseos) $\mathrm{y}$, con más frecuencia, es un lugar privado, a menudo adornado con figuras religiosas de diferentes orígenes, un incienso, una copa de agua y una vela. Nos parece que, como afirma Parker (2008), el sujeto religioso combina simbolismos diversos en una síntesis eminentemente sincrética, que satisface necesidades reales del hombre

\subsection{Los participantes}

En la consulta de tarot, los participantes del acto de intercambio comunicativo son el profesional que ofrece el servicio y la persona que consulta.

Para designar al profesional, se emplea tarotista, lector, intérprete, traductor, cartomántico, adivino y tarólogo.

La expresión tarotista se emplea en dos sentidos distintos. El primero se refiere "a persona que pretende predecir el porvenir por medio del tarot" (RAE, 2009); el segundo se refiere al profesional que entrega sugerencias y consejos (Fernández, 2007; Hales, 2000)

Peach (1989) lo denomina lector y le reconoce la cualidad de predecir de manera sensata y precisa; Bergman (1989) y Mayer (1996) hablan de intérprete, un tipo de médium -tal como se pensaba en la Edad Media- capaz de actuar como vehículo entre cartas y consultante; y Banzhaf (2005), traductor, ya que entiende el lenguaje de las imágenes y se las explica al oyente.

Jiménez del Oso (1980) y Cousté (1980) lo Ilaman adivino. El primer autor, por la capacidad de predecir, gracias a su facultad telepática innata; el segundo, porque puede imaginar con certeza el futuro. El término cartomántico igualmente designa a quien tiene como misión adivinar el futuro (Jiménez del Oso, 1980; Wirth, 1982; Salas, 1992).

8 Véase B. Gallardo (1998). 
El tarólogo, palabra acuñada por Jodorowsky (2004), lee el presente, que es lo realmente desconocido para el consultante, aun cuando este busque datos acerca de lo que cree es su futuro. Además, dice el tarotista, cualquier ser humano puede aprender a leer y traducir los mensajes del tarot (ser tarólogo) sin necesidad de ser un mago, un vidente, un ser dotado de poderes parapsicológicos.

La denominación tarotista es la preferida por la muestra. Para autodenominarse, también emplean los términos lector/a de cartas y bruja. El primero con la intención de diferenciarse de quienes leen con fines predictivos y el segundo vinculado con la posesión de poderes psíquicos.

Para denominar al no profesional, es decir, la persona que solicita la lectura de cartas, se emplea consultante, cliente, interrogador y paciente.

La palabra consultante es la más empleada por estudiosos del tarot (Irish, 1978; Cousté, 1980; Wirth, 1982; Nichols, 1989; Salas, 1992; Mayer, 1996; Gray, 1997; Hales, 2000; McCormack, 1999; Jodorowsky, 2004; Banzhaf, 2005; Thomson y otros, 2005; Matienzo, 2006; Fernández, 2007) y por los participantes de nuestra muestra. Jodorowsky (2004) emplea, además de consultante, el vocablo paciente, expresión empleada por quienes siguen el enfoque terapéutico.

El término interrogador muestra la faceta relacionada con plantear preguntas durante la sesión (Peach, 1989; Bergman, 1989). Por el hecho de contratar un servicio profesional, se le llama cliente (Nichols, 1989; Noble, 1991).

La relación entre ambos participantes es de desigualdad funcional. Cada uno desempeña su papel y no hay intercambio de estos roles. La asimetría se funda en el conocimiento del tarot del profesional quien, supuestamente tiene el poder de desvelar información oculta para el cliente. Ahora bien, a pesar del poder mayor del tarotista, en cuanto a su capacidad para interpretar las cartas, estratégicamente, este intenta aproximarse al consultante, mostrando solidaridad y disminuyendo, por tanto, esa asimetría de partida. Por ejemplo en (1), la tarotista para establecer cercanía con su cliente, se distancia de su rol de experta pues declara ser ignorante e imperfecta como todo ser humano:

(1) TM: uno no sabe SOMOH HUMANOH (...) TAN IMPERFECTOH ${ }^{9}$

\subsection{Dinamismo comunicativo. Alternancia de turno}

Hay un proceso de lectura planificado que se cumple de manera fija. No se pueden intercambiar los papeles de los participantes y la alternancia de turno está

9 La transcripción del corpus se ajusta al sistema Val.Es.Co. (Briz y Grupo Val.Es.Co., 2002). 
predeterminada. Sobre la toma de turno, el tarotista es quien inicia y cierra la sesión, y la "modera".

Basándonos en la propuesta de unidades del grupo de investigación Val.Es.Co (2014), en nuestra muestra identificamos intervenciones que evidencian el dinamismo comunicativo recomendado por algunos autores (Cousté, 1980; Matienzo, 2006). Así registramos intervenciones en que el tarotista emite actos reactivos como manifestaciones de acuerdo y aprobación, así como respuestas cooperativas; e intervenciones iniciativas directas, es decir, intervenciones que requieren habitualmente de una reacción explícita por parte del consultante.

Además, las respuestas del tarotista a menudo confirman los papeles comunicativos con reacciones como ya, $\mathrm{mhm}$. Así, el lector también permite de forma estratégica que el consultante sea protagonista, dejando que intervenga, escuchándolo. Por ejemplo, en (2), el lector responde a la consultante que media cargadita significa que ha sido objeto de brujería, demanda información acerca del origen del maleficio (¿con quién tuvo problemas?) y emite una respuesta fática (ya) que demuestra atención a la intervención reactiva de su interlocutora:

(2) TH: EHTÁ MEDIA CARGADITA

C: ya ¿a qué se refiere con media cargadita?

$\mathrm{T}$ : puede que ehtén haciendo cositah malah (...) BRUJERÍA se podría decir

C: ya

$\mathrm{T}$ : ¿con quién tuvo problemah?

C: con la persona que le dije nada máh

T: ya

\subsection{Planificación de la sesión}

La consulta de tarot es un evento planificado que presenta cuatro momentos claramente diferenciados: barajar, cortar, distribuir e interpretar. Además, a menudo se mantiene un diálogo antes de leer las cartas como mecanismo de acercamiento al cliente, consejo que realizan algunos autores (Irish, 1978; Salas, 1992).

De acuerdo con los análisis de nuestras muestras, distinguimos tres etapas que hemos llamado diálogos de inicio de sesión, diálogos de la sesión y diálogos de cierre.

a) Diálogos de inicio de sesión. En esta fase se recomienda indagar en los datos personales y el motivo de la consulta (Irish, 1978; Salas, 1992). En nuestra muestra, a menudo los profesionales consideran este consejo, ya que saben, en palabras de Perelman y Olbrechts-Tyteca (1989:56), que "el conocimiento (...) de aquellos cuya adhesión piensa obtener es, pues, una condición previa a toda argumentación eficaz".

En este diálogo, a menudo el tarotista describe aspectos positivos de la personalidad del cliente a partir de otros oráculos para ganar su simpatía y demostrar conocimiento 
esotérico. Por ejemplo, en (3), emplea adjetivos que valorizan el signo zodiacal del consultante, algunos pronunciados de manera enfática:

(3) TM: tauro (...) eh excelente $\uparrow$ EH FUERTE EH VALIENTE

Asimismo, el tarotista se esfuerza por poner de relieve aspectos que garantizan su credibilidad. Por ejemplo, en (4) rechaza la postura predictiva, pues plantea un destino prefijado, que destaca con el silabeo y un cambio de voz que expresan ironía en a di vi na to rioh:

(4) TH: no te voy a/ hablar/ en términoh típicoh llamémohle a di vi na to rioh

En nuestra muestra, también a menudo el lector informa la secuencia de los métodos. Por ejemplo, en (5), indica que la primera lectura es general y la segunda, de preguntas:

(5) TM: vamoh a ver primero una mirada general $\rightarrow$ /tú dehpuéh me preguntah

b) Diálogos de la sesión. En esta etapa se baraja y corta el mazo, se seleccionan las cartas, se distribuye el naipe e interpretan los símbolos.

Es habitual que el consultante baraje y corte el mazo al inicio de cada método. Así se persigue lograr sincronicidad para obtener respuestas más claras (Noble, 1991). Cuando pide barajar y cortar las cartas, el tarotista emplea con frecuencia fórmulas de cortesía que atenúan la orden. En (6), por ejemplo, usa una perífrasis verbal atenuante; y en (7), usa la construcción analítica hacer un corte con dativo de interés me, además sigue el consejo de pedir cortar con la mano izquierda para reflejar "el subconsciente" (McCormack, 1999:20):

(6) TM: primero te voy a pedir que revuelvah este mazo

(7) TH: con la mano ihquierda me hace un corte

Los símbolos se distribuyen, seleccionan e interpretan según el método libre, caracterizado por el empleo de una cantidad indeterminada de cartas sin una estructura y el método estructurado, procedimiento basado en el empleo de un número definido de cartas que se ordenan en un esquema predeterminado (Ramírez, s.f.). Los lectores de nuestra muestra aplican los métodos estructurados llamados tirada y pregunta/respuesta.

La tirada corresponde a un diagrama cuyas posiciones expresan un campo temáticotemporal. En nuestra muestra, por ejemplo, es frecuente una tirada temática de once cartas relativas al estado presente, la influencia del pasado en los hechos actuales y el futuro.

La pregunta/respuesta es una fórmula que se propone contestar una duda del cliente con un número limitado de cartas. En nuestro corpus, a menudo el tarotista emplea de tres a seis cartas y, cuando este método se acompaña de una tirada, su duración generalmente dobla la tirada, dato que revela la importancia de atender a las 
necesidades del consultante. Además, en nuestra muestra, con frecuencia las respuestas expresan una predicción, ya que es habitual que los consultantes soliciten una lectura sobre el desarrollo de un evento en un tiempo futuro.

Después de distribuir las cartas, el tarotista elabora diálogos de interpretación de los símbolos. Significa "unir el significado especial de cada carta con el significado del campo sobre el que está" (Banzhaf, 2005:59). En nuestra muestra, los campos temático-temporales más frecuentes, y expresados también por algunos autores (Cousté, 1980; Thomson y otros, 2005), son el mundo interno del consultante (emociones, creencias, etc.) y el desarrollo en diversas áreas (amor, trabajo, dinero, etc.), a menudo interpretados en pasado, presente y futuro. Además, como en (8) es habitual el anuncio en un campo no especificado:

(8) TM: eh un CAMBIO que va a ser pa' mejor

Respecto de la frecuencia con que se presenta un tema, el amor de pareja es el más solicitado por los clientes (Banzhaf, 2006), dato también expresado por profesores de tarot ${ }^{10}$ y constatado en el análisis del corpus. Por ejemplo, en (9) la consultante decide preguntar por el amor y argumenta que los otros ámbitos se resuelven sin ayuda:

(9) TM: ¿qué vah a preguntar?

C: me interesa la pareja (...) el rehto de lah cosah se resuelven

Para lograr la continuidad de la lectura, es habitual el uso preguntas retóricas, que, además de reafirmar lo que se está diciendo, se logra con estas atraer la atención del cliente. Por ejemplo, en (10), la pregunta retórica se refiere al pensamiento de la consultante:

(10) TM: ¿qué tieneh en la cabeza? (...) eh tu mundo material

Asimismo, el lector emplea habitualmente, para iniciar un método, formas indirectas con plural inclusivo que van guiando al cliente. Además, plantea preguntas para adecuar las respuestas a los datos y, así, ser más convincente. Por ejemplo, en (11) señala que corresponde el método de pregunta/respuesta; y en (12), la tarotista, antes de leer las cartas, pregunta por el pasado amoroso:

(11) TM: ahora vamoh a preguntar

(12) TM: ¿tú ehtabái con pareja?

Al final de la sesión, es frecuente que el tarotista formule una síntesis de la interpretación y emplee marcadores que indican el término de la lectura. Por ejemplo, en (13), usa un marcador que indica el fin de la lectura (eso), después de una conclusión positiva para el año siguiente. Con la entonación se destacan los mensajes que benefician al cliente: tieneh $\uparrow$; y la fecha anunciada: doh mil doce $\uparrow:$

(13) TM: tieneh $\uparrow$ el movimiento/el doh mil doce $\uparrow / v a$ a ser pa' ti un año ehpectacular///eso

10 Opiniones registradas en la observación participante de clases de tarot. 
c) Diálogos de cierre de sesión. Por lo general, en estos diálogos se concreta el pago de la sesión y el lector expresa interés por el bienestar del cliente. Por ejemplo, en (14) le confirma el valor de la sesión y le sugiere que use, por seguridad, joyas de poco tamaño:

(14) TH: dieh no máh (...) y la joya siempre pequeña/ no useh cosah grandeh

Los diálogos de inicio de sesión, los de sesión propiamente y de cierre se enmarcan entre otros dos diálogos, el de apertura y el de despedida. A menudo, el diálogo de apertura marca el comienzo del evento comunicativo con un saludo y el tarotista le indica al cliente que tome asiento junto a la mesa (hola/asiento); y en el diálogo de despedida, que señala el final del mismo, incluye fórmulas de buenos deseos (chao/ que te vaya bien).

\subsection{Finalidad de la sesión}

El tarotista elabora un mensaje para responder las dudas, posibles o manifiestas, del consultante en diversos campos temático-temporales. Basándose en el criterio de autoridad, se propone persuadir de la verdad de la lectura. Su actividad es, por tanto, argumentativa, pues plantea razones para llegar a una conclusión ${ }^{11}$.

Por otra parte, la lectura del tarot se basa en la creencia de distintas propiedades de los símbolos, que nuestro estudio ha resuelto en tres tipos: capacidad adivinatoria (Cousté, 1980; Waite, 1989), orientadora (Fernández, 2007; Escola Mariló Casals, 2013) y terapéutica, impulsada en la década de los noventa por Jodorowsky (2004). Así, la tarea será convencer al consultante sobre la realización futura de un hecho, la mejor solución a un problema o justificar la necesidad de sanar el inconsciente.

En la lectura de cartas de nuestra muestra, las finalidades adivinatoria y orientadora son habituales, aunque los tarotistas declaran rechazar la idea de anticipar el futuro. La visión terapéutica no se presenta, pues las lecturas relativas a la sanación registran sobre todo fines predictivos y orientadores.

A menudo la actividad argumentativa se inicia con la lectura de un diagnóstico negativo con frecuencia realzado, para así destacar las soluciones que se van a ofrecer. Por ejemplo, en (15) el tarotista realza prosódicamente que la consultante está embrujada:

(15) TH: ehtá BIEN CARGADITA/ ALGUIEN LA EHTÁ CARGANDO

Además, es habitual que una predicción auspiciosa se elabore sin determinación temporal y de manera intensificada, especialmente al finalizar la lectura del tema. Por

11 Como señalan Portolés y Yate (2014), esta es la definición derivada de la mayoría de los estudios contemporáneos que describen la construcción argumentativa del discurso (Perelman \& Olbrechts - Tiyteca, 1969; Toulmin, 1958; Plantin, 1996; Lo Cascio, 1991; Van Eemeren; Grootendorst \& Snoek Henkermans, 2002; Marraud, 2007). 
ejemplo, en (16), el tarotista emplea la pronunciación marcada para realzar un cambio de etapa y adverbios en grado superlativo que destacan la seguridad del presagio:

(16) TH: va a pasar a otra etapa/ Y UN CICLO QUE MUERE Y UN CICLO QUE COMIENZA

(...) le va a ir muy bien/ superbién

En relación con la finalidad orientadora, es frecuente que el tarotista imprima fuerza argumentativa a lo dicho por medio de continuas repeticiones y una pronunciación enfática, Por ejemplo, en el caso de (17) el tarotista destaca el consejo no te quedes ahí, lo cual significa que debe seguir estudiando:

(17) TM: NO TE QUEDEH AHÍ/NO TE QUEDEH CON ESO/no te quedeh ahí

Además es habitual que, para evitar imponer soluciones, los lectores sustituyan enunciados obligativos (tienes que o debes) por una construcción más abierta y atenuada de posibilidad. Por ejemplo, en (18), la tarotista señala una recomendación que atañe a la pareja: podría tratar de conversar con él para que consulte un sicólogo:

(18) TM: podríah tratar de conversar que/mm /que vaya al sicólogo $\uparrow$ ¿ah?

Respecto del criterio de autoridad, en nuestro corpus, la carta y el propio tarotista son las fuentes de verdad para expresar adivinaciones y orientaciones. Con frecuencia, están presentes en la enunciación de un mismo campo temático-temporal. Por ejemplo, en (19) se basa en el símbolo de El Mago para predecir la venta de una casa y muestra adhesión a la predicción (yo creo), en la cual ella se muestra como fuente de verdad:

(19) TM: la vah a vender rápido porque te va a salir El Mago allí/ entonceh yo creo/ que junto con vender esa casa TÚ CIERRAH UN CAPÍTULO/

También es frecuente que la autoridad se enuncie de manera impersonalizada. EI tarotista así minimiza su responsabilidad como lector. Por ejemplo, en (20), el tarotista enuncia que le sale La Estrella, argumento para concluir un futuro positivo:

(20) TH: le va a ir muy bien/ le sale La Ehtrella

Como el tarotista pretende persuadir de la verdad del mensaje con distintos fundamentos, es frecuente la adición de argumentos coorientados. Por ejemplo, en (21), la tarotista asegura que en el presente no debe decidir. Se basa en la predicción de que tendrá claridad para resolver y en la ayuda simbolizada por la carta llamada Ángel Custodio:

(21) TM: no eh tiempo pa' tomar ninguna decisión//porque vai a tener la claridá sobre una decisión que te ordena/y que ademáh $\uparrow$ es una decisión supeerbonita $\uparrow$ porque viene cuhtodiada //te sale el Ángel

En cuanto a los tópicos argumentativos, se observa una relación directa entre un estado de felicidad personal con tener pareja, trabajo y dinero. Por ejemplo, en (22), la tarotista presagia que la consultante celebrará una nueva unión amorosa:

(22) TM: va a aparecer alguien en tu vida// (...) porque te veo como celebrando 
Asimismo, en general, la vaguedad de los mensajes caracteriza la actividad argumentativa. Moulian (1997:142) afirma que el agente mágico emplea principios comunicacionales de enorme efectividad, tales como "contenidos abiertos y ambiguos, que exigen una interpretación desde la experiencia y el contexto del destinatario para adquirir pleno sentido". Por ejemplo, en (23) se frecen varias posibilidades siempre de forma atenuada mediante el empleo de formas deícticas vagas, expresiones que hacen borroso el significado (ahí, algo así, como), verbos de posibilidad y formas temporales condicionales (podría ser), para evitar responsabilidad sobre lo informado, lo que se intenta a su vez apelando al otro para que confirme lo expresado ( $¿ y a, ~ ¿ m m$ ?). Además la expresión de la temporalidad del suceso y del hecho mismo son ambiguos: está en espera de sanación o a punto de sanarse; puede ser triángulo o algún tipo de indecisión:

(23) TM: hay un tema amoroso ahí como triangular/que ehtá ahí// en ehpera de sanación ¿ya?//o que ehtá a punto de sanarse algo así $\downarrow$ o que se está mirando para sanarse ¿ya? no sé si puede ser triángulo pero también podría ser//algún tipo de indecisión de de no saber si ehtar con esa persona $\rightarrow$ o no ¿mm?

\subsection{Registro lingüístico}

En relación con la modalidad lingüística o registro empleado, en los mensajes estudiados se combina un tono semiformal a veces y coloquial otras: los tarotistas emplean tanto expresiones técnicas, propias de un género que es profesional, como coloquiales. Por ejemplo, en (24), la tarotista para explicar cómo se desarrollará la crisis en el futuro, emplea la expresión propia del registro coloquial "hacia dónde va la micro":

(24) TM: hay unah cartah que son de crisih $\rightarrow$ de no tener claridad respecto de hacia dónde va la micro

Asimismo, coloquial es la sintaxis y el modo de articular y de hacer que progrese el discurso. Se presentan saltos temáticos, alto grado de redundancia y secuencias sin uso de marcadores, hecho más frecuente en tarotistas de menor instrucción; es decir, un progreso de la información que se presenta sobre la marcha, lo que es más propio de lo oral coloquial. Por ejemplo, en (25), se logra que el discurso progrese y esté cohesionado gracias al empleo del conector continuativo $y$, así como de estructuras enumerativas; en efecto, el tarotista anuncia el fin de un ciclo, un viaje, la llegada de visitas, que está embrujado el trabajo; repite la llegada de visitas:

(25) TH: como que ehtá culminando una etapa y va a pasar a otra etapa/ Y UN CICLO QUE MUERE Y UN CICLO QUE COMIENZA/ VA A HACER UN VIAJE / tiene la llegada de visitah/ EL JARDÍN EHTÁ CARGADO/viene gente de afuera VA A VENIR UN MATRIMONIO DE VISITA

\section{CONCLUSIÓN}

La consulta de tarot es una interacción que en Santiago de Chile se presenta habitualmente en la oferta de servicios esotéricos. El interés actual hacia esta práctica 
se origina en una nueva religiosidad que busca respuestas a problemáticas internas del sujeto. Según nuestro estudio y datos gubernamentales, la creencia en el tarot cuya lectura es legal desde 2003- está más arraigada en mujeres con instrucción superior.

Respecto de los rasgos situacionales, el evento oral analizado se desarrolla cara a cara en un marco de interacción no cotidiano, la consulta, espacio donde se dispone de ornamentación que demuestra sincretismo cultural. A menudo, el lector prefiere interpretar un tarot con diseño tradicional en un tiempo cercano a una hora.

Las denominaciones tarotista y consultante son las formas prototípicas que designar a los participantes. También registramos que la selección de un término para nombrar al profesional puede responder a la función predictiva, orientadora o terapéutica que se le atribuye al tarot, además de mostrar cualidades del intérprete. Cada integrante desempeña su rol: el tarotista emplea el poder que le entrega el conocimiento especializado y el consultante recibe esa información.

La mayor planificación previa se manifiesta claramente en una estructura que presenta diálogos marco, diálogos de inicio de sesión, propiamente de sesión y de cierre. Asimismo, en los diálogos de la muestra predominan secuencias constituidas por una sucesión de intercambios que reflejan el carácter retroalimentado del evento. Así, se logra un encuentro colaborativo, en el cual es posible una mayor cercanía con el consultante.

En general, el diálogo de inicio tiene características de exordio, porque el orador se esforzará por mostrar su credibilidad y honestidad para convencer de la verdad de los mensajes. Habitualmente, también valoriza la imagen del cliente para ganar su simpatía.

Estructuralmente, en los diálogos de la sesión a menudo se pretende lograr el efecto mágico que se atribuye al contacto previo a la interpretación. La lectura de las cartas se realiza de acuerdo con un método estructurado, tirada y pregunta/respuesta, sobre diversos campos temático-temporales, a pesar del rechazo manifestado por los tarotistas hacia la lectura predictiva.

A menudo el tarotista emplea recursos de construcciones de transición entre temas y métodos, con el fin de mantener la atención del consultante y obtener datos para una lectura más persuasiva. También con frecuencia la interpretación incluye un resumen de la lectura y, en el cierre de sesión, se concreta el pago de la sesión y el lector enuncia un mensaje que demuestra interés hacia el cliente.

Respecto de la actividad argumentativa, en la muestra es habitual que la lectura presente las finalidades adivinatoria y orientadora. Se produce, entonces, una 
disonancia cognitiva, pues los lectores, aunque declaran rechazar la idea de anticipar el futuro, acompañan los consejos con predicciones.

Generalmente, las conclusiones argumentativas se expresan en una temporalidad indeterminada, anuncian cambios auspiciosos que dan esperanza al cliente y se formulan evitando imponer soluciones. Asimismo, con el fin de persuadir de la veracidad del mensaje, el tarotista a menudo combina diferentes recursos intensificadores para ofrecer seguridad al consultante, todo lo cual imprime fuerza argumentativa a lo dicho.

Además, en el corpus estudiado, el símbolo y el tarotista se constituyen en fuentes de verdad, en argumentos de autoridad, con el propósito de dar respuesta a la problemática del consultante. No obstante, también es frecuente que no se identifique la autoridad y se enuncie de manera impersonalizada para evitar responsabilidad sobre la interpretación e imprimir mayor objetividad a la lectura.

Como el tarotista de nuestro estudio pretende persuadir de la verdad de los hechos con distintos fundamentos, es frecuente la coorientación. En cuanto a los tópicos argumentativos, se observa una relación directa entre un estado de felicidad con tener pareja, trabajo y dinero. Finalmente, el estudio de nuestra muestra refleja que, en general, el mayor fin transaccional del discurso, materializado en mensajes expresados de manera vaga, fácilmente adaptables a cualquier problemática, se combina con un tono semiformal a veces y coloquial otras.

La descripción realizada ha dado cuenta del estudio de consultas cuya actividad argumentativa del tarotista aborda tanto el diagnóstico de la situación del consultante como la elaboración de soluciones y predicciones. Por ello, consideramos que nuevos análisis podrían investigar consultas en las cuales la solución a una situación negativa se encuentra en la ejecución de un servicio complementario. Nos referimos, en especial, a consultas en que, además de persuadir de la verdad de la lectura de cartas, el tarotista debe convencer al cliente de la necesidad de contratar trabajos adicionales hoy presentes en la oferta esotérica, como magia negra y psicomagia.

\section{REFERENCIAS BIBLIOGRÁFICAS}

Agar, Lorenzo (2011): «Migraciones posmodernas en Chile: Reflexiones sobre cohesión social y pluralidad cultural", Revista Palobra: Palabra que obra, 12, 108-129 [en línea]:

http://ojs.udc.edu.co/index.php/palobra/articl e/view/119/106. [Consulta: 12/12/2013].

Bahamondes, Luis (2000): «Una mirada a la metamorfosis religiosa en América Latina: nuevas ofertas de sentido en la sociedad contemporánea», Revista Científica Guillermo de Ockham. 10 (2), 109-116 [en línea]:

https://www.academia.edu/5007175/Baham ondes_L._2012_._Una_mirada_a_la_meta morfosis_religiosa_en_Am\%C3\% $\overline{\mathrm{A}}$ 9rica_Lat ina_nuevas_ofertas_de_sentido_en_la_soci edad_contempor\% $\overline{\mathrm{C}} 3 \% \overline{\mathrm{A}} 1 \mathrm{nea}$. [Consulta: 12/12/2013].

Banzhaf, Hajo (2005): Las llaves del tarot, Madrid, Edaf.

Banzhaf, Hajo (2006): Tarot: una guía de ayuda para tomar decisiones, Madrid, Edaf. 
Bergman, Klaus (1989): El poder del tarot, Madrid, Distribuciones Mateos.

Briz, Antonio (2010): «El registro como centro de la variedad situacional. Esbozo de la propuesta del grupo Val.Es.Co. sobre las variedades diafásicas», en Fonte, Irene y Lidia Rodríguez, coords., Perspectivas dialógicas en estudios del lenguaje. México, Editorial de la Universidad Autónoma de Nuevo León, 21-56 [en línea] http://www.valesco.es/web/Val.Es.Co/Miem bros/Antonio\%20Briz/Antonio\%20Briz/M\%C 3\%A9xico/la\%20propuesta\%20Valesco\%20 .pdf. [Consulta: 15/6/2014].

Briz, Antonio y Grupo Val.Es.Co. (2002): Corpus de conversaciones coloquiales, Anejo I de la revista Oralia, Madrid, Arco Libros.

Conicyt (2016): «Encuesta Nacional de Percepción Social de la Ciencia y Tecnología en Chile» [en línea]: http://www.conicyt.cl/blog/2016/07/conicytpresenta-resultados-de-la-encuestanacional-depercepcion-social-de-la-cienciay-la-tecnologia-en-chile/. [Consulta: 4/4/2017].

Cousté, Alberto (1980): El tarot o la máquina de imaginar, Barcelona, Bruguera.

Escola Mariló Casals (2013): Código ético del tarot [en línea] http://escolamarilocasals.wixsite.com/eticayt arot. [Consulta: 2/2/2014].

Fernández, Jimena (2007): Curso práctico de tarot, Barcelona, Océano Ámbar.

Gallardo, Beatriz (1998): Comentario de textos conversacionales II. Los textos, Madrid, Arco-Libros.

Gray, Eden (1997): Guía completa para el tarot, México, Diana

Grupo Val.Es.Co (2014): «Las unidades del discurso oral», Estudios de Lingüística del Español, 35, 13-73 [en línea] http://roderic.uv.es/bitstream/handle/10550/ 43945/102758. pdf? sequence $=1$ \&isAllowed= y. [Consulta: 12/9/2015]

Hales, Jaime (2000): El tarot: senderos de sabiduría, Santiago, Ediciones Tayta.

Injuv (2006): Quinta Encuesta Nacional de Juventud, Santiago, injuv.

Injuv (2009): Sexta Encuesta Nacional de Juventud, Santiago, injuv.

Irish, Joss (1978): El tarot. La baraja profética, México, Petronio.

Jiménez del Oso, Fernando (1980). El tarot: el futuro de las cartas, Madrid, Ediciones UVE.
Jodorowsky, Alejandro (2004): La vía del tarot, Buenos Aires, Sudamericana.

Matienzo, Adriana (2006): Tarot: significados, tiradas y más, Lima, Mirbet.

Mayer, Hugo (1996): Tarot: significados, tiradas y más, Barcelona, Editorial de Vecchi.

McCormack, Kathleen (1999): Tarot: orígenes, sistemas de lecturas e interpretación, Colonia, Taschen Benedikt.

Moulian, Rodrigo (2000): «Magia y Terapia Ritual: Principios Comunicacionales para conducir el cambio cognitivo», Revista Austral de Ciencias Sociales, 4, 133-150, 10.4206 /rev.austral.cienc.soc.2000.n4-08.

Nichols, Sallie (1989): Jung y el Tarot, Barcelona, Kairós.

Noble, Vicki (1991): Madre Paz, Santiago, Cuatro Vientos.

Parker, Cristián (2008): «Mentalidad religiosa post-ilustrada: creencias y esoterismo en una sociedad en mutación cultural», en Aurelio Alonso, comp., América Latina y el Caribe. Territorios religiosos y desafíos para el diálogo, Buenos Aires, clacso, 337-364 [en línea]:

http://bibliotecavirtual.clacso.org.ar/ar/libros/ grupos/alonso/Gumucio.pdf. [Consulta: 9/12/2013].

Peach, Emily (1989): Manual práctico del tarot, Madrid, Edaf.

Perelman, Chaïm y Lucie Olbrechts-Tyteca (1989): Tratado de la nueva argumentación, Madrid, Gredos.

Portolés, José y Jean Yates (2014): «The Theory of Argumentation Within Language and its Application to Discourse Analysis», en Casado, Manuel, Óscar Loureda e Inés Olza, eds., Use in the Public Sphere: Methodological Perspectives and Empirical Applications, Frankfurt, Peter Lang, 201-224, http://dx.doi.org/10.3726/978-3-0351-0526-1

Ramírez, Mariela: «Tiradas del Tarot» [en línea]: http://www.enbuenasmanos.com/esoterismo lel-tarot/tiradas-del-tarot/.

[Consulta: 23/4/2014].

Real Academia Española (2009): Diccionario de la lengua española, España, Espasa Calpe.

Salas, Emilio (1992): El gran libro del tarot, Barcelona, Hermética.

Serplac (2011): Evolución de indicadores de educación en la Región Metropolitana de Santiago: resultados encuestas CASEN 1990-2009, Santiago [en línea]: http://www.ministeriodesarrollosocial.gob.cl/ 
pdf/upload/2 INDICADORES EDUCACloN

_RMS_CASEN.pdf. [Consulta: 2/1/2015].

Thomson, Sandra y otros (2005): El corazón del tarot, Madrid, Arkano Books.
Waite, Arthur (1989): Claves del tarot. El tarot Rider- Waite, Barcelona, Obelisco Adivinación.

Wirth, Oswald (1982): El tarot de los imaginarios de la Edad Media, Barcelona, Archivo Esotérico. 